\title{
Prevalence of plasmid-mediated AmpC in Enterobacteriaceae isolated from humans and from retail meat in Zagazig, Egypt
}

Katrijn L. Rensing ${ }^{1 *}\left(\mathbb{D}\right.$, H. M. Abdallah², Alex Koek¹, Gamal A. Elmowalid², Christina M. J. E. Vandenbroucke-Grauls ${ }^{1}$, Nashwan al Naiemi, ${ }^{3,4}$ and Karin van Dijk'

\begin{abstract}
Background: The objective of this study was to determine the prevalence of plasmid-mediated AmpC (pAmpC) among Enterobacteriaceae isolated from humans and from retail meat in Egypt.

Methods: Enterobacteriaceae were isolated from patients with suspected bloodstream infection, human fecal samples, retail chicken meat samples and retail sheep meat samples. All group I Enterobacteriaceae were analyzed for presence of pAmpC genes by PCR. Antibiotic susceptibility testing was performed in all pAmpC positive isolates, followed by phenotypic and genotypic ESBL and carbapenemase testing on indication.

Results: The prevalence of pAmpC among group I Enterobacteriaceae isolated from 225 patients with bloodstream infection was 5.6\% [95\% Cl 2.2-13.4]. Among 100 patients with community-onset gastroenteritis the prevalence in fecal samples was 4.8\% [95\%Cl 2.1-10.7]. The prevalence among 112 chicken carcasses and 100 sheep meat samples was $2.4 \%$ [95\% Cl 0.7-8.4] and 1.1\% [95\%Cl 0.2-5.7], respectively. In half of the AmpC positive isolates we detected an ESBL gene and 2 isolates harbored a carbapenemase gene. In five isolates there was resistance to at least three important alternative antibiotic drugs.
\end{abstract}

Conclusions: We consider the prevalence of $\mathrm{pAmpC}$ in Egypt, as found in our study, moderately low. To follow future trends in prevalence of $\mathrm{pAmpC}$ worldwide, a standardized screening algorithm for the detection of PAmpC is needed.

Keywords: Plasmid-mediated AmpC, Group I Enterobacteriaceae, Prevalence, Egypt

\section{Background}

Resistance of Enterobacteriaceae to third generation cephalosporins has become a worldwide problem [1]. The most common cause of resistance to third generation cephalosporins is production of extendedspectrum beta-lactamases (ESBLs). The production of another class of beta-lactamases, the AmpC beta-lactamases (AmpC), also contributes to this problem [2]. This class of beta-lactamases has received less attention initially, but is now increasingly recognized as a growing problem in Enterobacteriaceae.

\footnotetext{
*Correspondence: k.rensing@vumc.nl; k.l.rensing@comicro.nl

${ }^{1}$ Amsterdam UMC, Vrije Universiteit Amsterdam, Medical Microbiology and Infection Control, Amsterdam Immunity and Infection Institute, Amsterdam, The Netherlands

Full list of author information is available at the end of the article
}

In several species of Enterobacteriaceae, AmpC is encoded on the chromosome. These species belong to the so-called group II Enterobacteriaceae (like Enterobacter spp., Serratia spp., Providentia spp., Morganella morganii, Citrobacter freundii and Hafnia alvei) [3]. In these species, chromosomally encoded AmpC can be transcribed at high levels by mechanisms such as induction and derepression, resulting in resistance to third generation cephalosporins [4]. Interestingly, AmpC can also be encoded on plasmids. Plasmid-mediated AmpC (pAmpC) genes are derived from chromosomal AmpC genes of group II Enterobacteriaceae and were initially found in Enterobacteriaceae that lack or have low expression of chromosomally encoded AmpC (like Klebsiella spp., Proteus spp., Salmonella spp., Escherichia coli, and Shigella spp.), so-called group I Enterobacteriaceae [2, 3].

(c) The Author(s). 2019 Open Access This article is distributed under the terms of the Creative Commons Attribution 4.0 International License (http://creativecommons.org/licenses/by/4.0/), which permits unrestricted use, distribution, and reproduction in any medium, provided you give appropriate credit to the original author(s) and the source, provide a link to the Creative Commons license, and indicate if changes were made. The Creative Commons Public Domain Dedication waiver (http://creativecommons.org/publicdomain/zero/1.0/) applies to the data made available in this article, unless otherwise stated. 
pAmpC may represent a new threat since plasmidencoded beta-lactamases are easily transferable between species and can cause nosocomial outbreaks [5-11]. Furthermore, the presence of $\mathrm{pAmpC}$ is often associated with multidrug resistance, and moreover, $\mathrm{pAmpC}$ in combination with porin loss may lead to resistance to carbapenems [5, 12-15]. Consequently, infections caused by pAmpC-producing Enterobacteriaceae have high therapy failure and mortality rates $[5,16]$.

The presence of pAmpC-producing Enterobacteriaceae in humans has been reported worldwide, also from several Mediterranean countries, like Spain [17, 18], France [19], Libya [20], Algeria [21], Morocco [22] and Turkey [23]. In addition, pAmpC has been detected in retail meat samples in Mediterranean countries and there are indications that retail animals might form a reservoir for $\mathrm{pAmpC}$-producing Enterobacteriaceae that could be transmitted to humans [24-27].

To date, little is known on the frequency of pAmpC in Egypt. Therefore, the aim of this study was to determine the prevalence of pAmpC among Enterobacteriaceae isolated from humans and from retail meat in Zagazig, Egypt.

\section{Methods}

\section{Sample collection and bacterial isolation}

Bacterial isolates from human bloodstream infections, human fecal samples, retail chicken meat samples and retail sheep meat samples were collected between January 2013 and May 2013, in Zagazig, Egypt. Complete sample collection and bacterial isolation have been described previously [28-30]. Below a summary is provided.

\section{Human blood samples}

Blood culture samples were obtained from 225 patients with a suspected blood stream infection at El-Ahrar General Hospital, Zagazig, Egypt. In case of growth of Enterobacteriaceae, one isolate per patient was included for further analysis [30].

\section{Human fecal samples}

Fecal samples were collected from 100 consecutive patients, who presented at El-Ahrar General Hospital, with community-acquired gastro-enteritis. This was defined as community onset of complaints of gastroenteritis and no prior hospital admission within the previous 10 days before start of the symptoms. Fecal samples (one per patient) were cultured on MacConkey agar [28].

\section{Retail sheep meat samples}

For a period of 10 weeks, two random meat samples were purchased each week at 5 retail butcher shops in different districts of Zagazig. The samples were immediately transported to the laboratory for culture. Sampling was performed by a swabbing-based method [31]. After collection, each swab was immersed in $5 \mathrm{~mL}$ of saline $(0.9 \%)$, mixed well by vortexing for 10 seconds, and centrifuged at 3,500 $\times \mathrm{g}$ for 15 minutes. Most of the supernatant was decanted, and $100 \mu \mathrm{L}$ of the sediment was inoculated directly on MacConkey agar.

\section{Retail chicken meat samples}

In seven butcher shops, located in different districts of Zagazig City, two random fresh chicken carcasses were bought once a week for a period of 8 weeks. Samples of these carcasses were cultured on MacConkey agar [29].

\section{Bacterial identification}

All morphologically different colonies were identified by the automated Vitek $^{\circ}$ MS system (BioMérieux, Marcy l'Étoile, France). After identification, Enterobacteriaceae were divided into two groups: group I Enterobacteriaceae, in which inducible or derepressed chromosomal AmpC enzymes are uncommon or absent (like Escherichia coli, Klebsiella spp., Proteus mirabilis, Salmonella spp., and Shigella spp); and group II Enterobacteriaceae, in which inducible or derepressed chromosomal AmpC is common (like Citrobacter freundii, Enterobacter spp., Morganella morganii, Serratia spp., Hafnia alvei and Providencia spp.). Two vials of cultured Enterobacteriaceae were lost: one (R.ornithinolytica) in the group of patients with gastroenteritis and one (E.coli) in the group of chicken meat samples. Therefore, these two isolates are not included in the analysis.

\section{AmpC PCR}

All group I Enterobacteriaceae were analyzed by PCR for molecular detection of $\mathrm{pAmpC}$ genes. For this purpose we up-dated the AmpC PCR that was published by Brolund et al. in 2010 [32]. This PCR was based on 2 triplet PCR reactions followed by melting point analyses to differentiate between genes belonging to the 6 different AmpC families (CIT, MOX, FOX, ACC, DHA and EBC), as described by Pérez-Pérez [33]. We adapted the primer set in order to also detect more recently described AmpC genotypes (Table 1). The thermal cycling conditions were: $95^{\circ} \mathrm{C}$ for 10 minutes, followed by 35 cycles of $95^{\circ} \mathrm{C}$ for 15 seconds and $65^{\circ} \mathrm{C}$ for 1 minute. Dissociation conditions were $95^{\circ} \mathrm{C}$ for 1 minute, followed by ramping started at $60^{\circ} \mathrm{C}$ for 1 minute continuously increasing towards $95^{\circ} \mathrm{C}$.

Antibiotic susceptibility testing and confirmation of ESBLand carbapenemase production

Antibiotic susceptibility testing was performed for all pAmpC PCR positive Enterobacteriaceae by the automated VITEK $^{\circ} 2$ system with AST card N198 or N344 (BioMérieux, Marcy l'Étoile, France). The MIC breakpoints according to the EUCAST criteria were used for 
Table 1 Overview of primers

\begin{tabular}{lll}
\hline Primers & Sequence 5'-3' $^{\prime}$ & Melting point \\
\hline CIT-F1 & TGG CCA GAA CTG ACA GGC AAA & $87.84^{\circ} \mathrm{C}$ \\
CIT-F2 & TGG CCT GAA CTG ACT GGT AAG & \\
CIT-R1 & TाT CTC CTG AAC GTG GCT GGC & \\
CIT-R2 & TाT CTC CTG AAC GCG GCT GGC & \\
CIT-R3 & TाT CTC CTG AAC CTG GCT GGC & \\
MOX-F & GCT GCT CAA GGA GCA CAG GAT & $91.04^{\circ} \mathrm{C}$ \\
MOX-R1 & CAC ATT GAC ATA GGT GTG GTG C & \\
MOX-R2 & CAC ATT GAG GTA GGT ATG GTA C & \\
FOX-F & AAC ATG GGG TAT CAG GGA GAT G & $89.15^{\circ} \mathrm{C}$ \\
FOX-R & CAA AGC GCG TAA CCG GAT TGG & \\
DHA-F & AAC TIT CAC AGG TGT GCT GGG T & $88.23^{\circ} \mathrm{C}$ \\
DHA-R & GCT GCC ACT GCT GAT AGA A & \\
ACC-F & GTG CAA GCC AAT ATG GGG CAG & $83.58^{\circ} \mathrm{C}$ \\
ACC-R & CTC CCA CAT CAG ATC CTG AGT & \\
EBC-F1 & TCG GTA AAG CCG ATG TTG CGG & $89.50^{\circ} \mathrm{C}$ \\
EBC-F2 & TTG GCA AAG CCG ATA TCG CGG \\
EBC-F3 & TTG GCA AGG CCG ATA TCG CGG \\
EBC-R1 & CTT CCA CTG CGG CTG CCA GTT \\
EBC-R2 & CGC CCA CTG TGG TTG CCA GGA \\
EBC-R3 & CTT CCA CTG CGG TTG CCA GTG & \\
\hline
\end{tabular}

interpreting the results [34]. ESBL- and carbapenemase production were phenotypically confirmed according to the EUCAST guideline for the detection of resistance mechanisms [35] and the guidelines of the Dutch Society of Medical Microbiology [3], using the ESBL combination disk (Rosco, Taastrup, Denmark) and carbapenemase double disc synergy test [36, 37], respectively.

All phenotypic ESBL- and carbapenemase positive isolates were analysed for the presence of genes encoding bla $a_{\mathrm{TEM}}, b l a_{\mathrm{SHV}}$ and $b l a_{\mathrm{CTX}-\mathrm{M}}$, and/or bla $a_{\mathrm{KPC}}, b l a_{\mathrm{NDM}}$, $b l a_{\mathrm{OXA}-48}, b l a_{\mathrm{IMP}}$ and $b l a_{\mathrm{VIM}}$, as previously described [28]. If $b l a_{\mathrm{TEM}}$ or $b l a_{\mathrm{SHV}}$ encoding genes were present in the absence of bla $a_{\mathrm{CTX}-\mathrm{M}}$, purified PCR products were sequenced for further analysis [28]. In case of carbapenemase production, and phenotypic ESBL testing could not be interpreted, genotypic ESBL testing was performed, as recommended by EUCAST [35].

\section{Statistical analysis}

Results were expressed as the proportion of group I Enterobacteriaceae that were pAmpC positive. Confidence intervals were calculated using Wilson's score. Analyses were performed with $\mathrm{R}$ package version 3.5.0.

\section{Results}

We isolated 94 Enterobacteriaceae from 225 patients with suspected blood stream infection. Seventy-two were group I Enterobacteriaceae of which four were positive in the AmpC PCR [5.6\% (95\%CI 2.2-13.4)] (Fig. 1 and Table 2). pAmpC was detected in three E.coli strains and one K.pneumoniae strain. In 100 fecal samples of patients with community-onset gastroenteritis we detected five pAmpC positive isolates out of 105 group I Enterobacteriaceae
[4.8\% (95\%CI 2.1-10.7)]. In this patient group, pAmpC was found in three K.pneumoniae strains and two P.mirabilis strains, all derived from distinctive patients. Overall, three out of 127 clinical E.coli isolates $(2,4 \%)$ and four out of 38 clinical K.pneumoniae isolates (10.5\%) were pAmpC positive.

Of the 112 chicken carcasses we collected, cultures of 12 carcasses had to be discarded because of growth of Pseudomonas spp. $(n=7)$ or Gram-positive cocci $(n=5)$. In the remaining 100 chicken carcasses, there was growth of 83 group I Enterobacteriaceae, among which two were pAmpC positive [2.4\% (95\%CI 0.7-8.4)]. In sheep meat, one E.coli isolate out of 95 group I Enterobacteriaceae was AmpC positive (1.1\% (95\%CI 0.2-5.7).

AmpC variants belonging to the CIT family were found most often $(n=9)$ followed by variants belonging to DHA family $(n=2)$ and EBC family $(n=1)$.

Table 3 shows susceptibility patterns and presence of ESBL and/or carbapenemases in all $12 \mathrm{pAmpC}$ encoding group I Enterobacteriaceae. In half of the isolates we detected an ESBL gene. Two isolates were phenotypically ESBL positive, which could not be confirmed genotypically. Two isolates showed carbapenemase production and harbored $b_{l} a_{\mathrm{VIM}}$. Five out of $12 \mathrm{pAmpC}$ encoding Enterobacteriaceae showed resistance to three out of the following antimicrobial categories: aminoglycosides, quinolones, carbapenems and trimethoprim/sulfamethoxazole.

\section{Discussion}

The prevalence of pAmpC in group I Enterobacteriaceae isolated from humans and from retail meat in Egypt varied between $1 \%$ and $6 \%$. Nearly $5 \%$ of Egyptian patients with community-onset gastroenteritis were colonized with pAmpC-producing group I Enterobacteriaceae. In patients with suspected blood stream infection, the prevalence of pAmpC among group I Enterobacteriaceae isolated from blood was comparable. In contrast, Helmy et al. found a prevalence of pAmpC of 16\% among group I Enterobacteriaceae that were isolated from Egyptian hospitalized patients with a urinary tract infection [38]. This higher prevalence might be explained by a difference in patient populations: Helmy et al. collected samples from hospitalized patients, including ICU patients, whereas in our study samples were derived from both hospitalized and non-hospitalized patients. As suggested by Helmy et al., the hospitalized patients in their study may have recently been exposed to cephalosporin therapy, which has been described as an independent risk factor for infection with pAmpC-producing Enterobacteriaceae [39]. Another study that has been conducted in Egypt, among healthcare workers of an Egyptian hospital, showed a prevalence of $3 \%$ : out of 200 health care workers, six were colonized with pAmpC producing E.coli [40]. As a reference, in other North African countries, prevalence of $\mathrm{pAmpC}$ in humans 


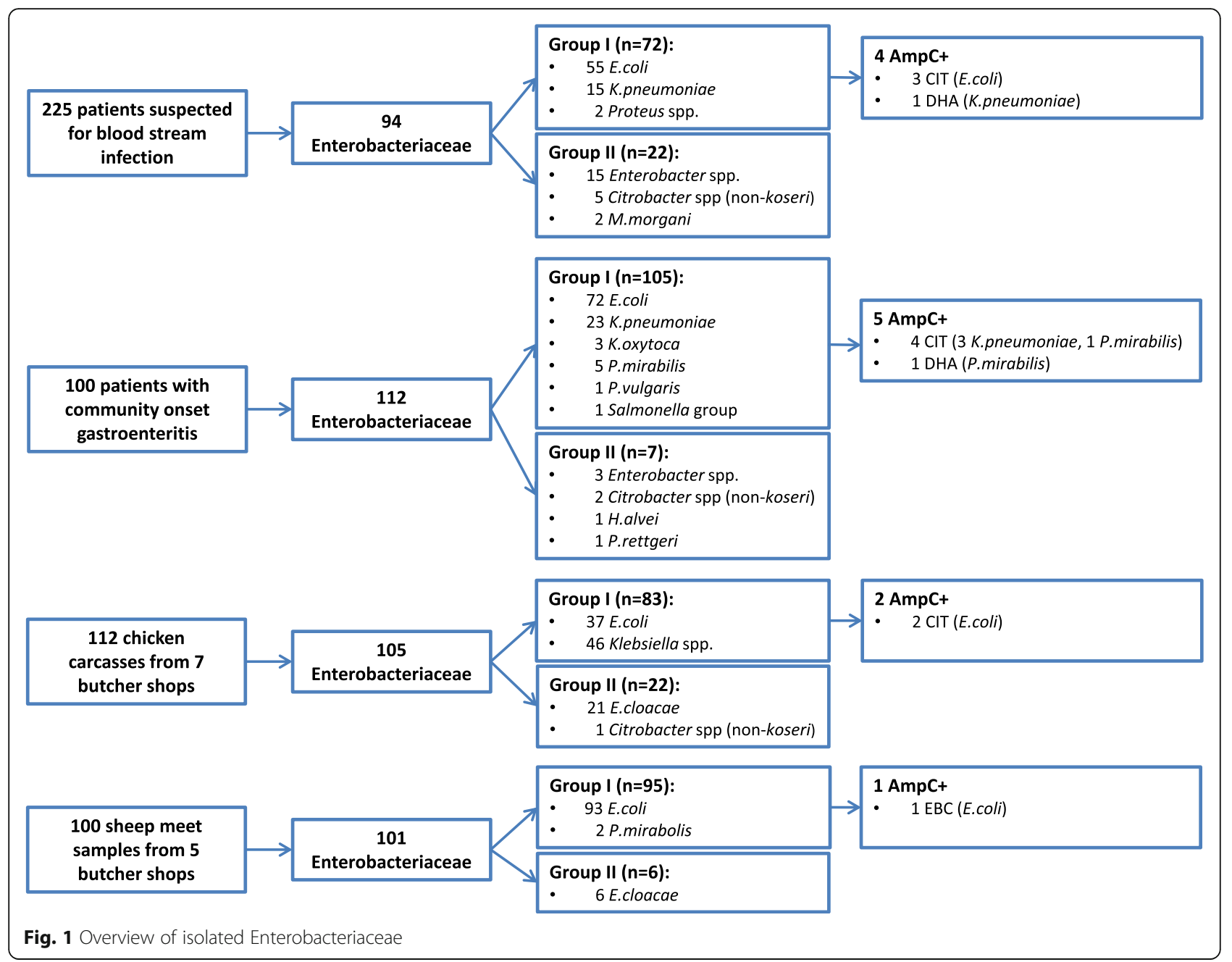

varied between 2.3\% of Enterobacteriaceae in Algeria [21] and $6.7 \%$ of E.coli in Libya [20].

In Europe, the prevalence of pAmpC seems a bit lower than the prevalence we found in Egypt. In the Netherlands, the prevalence among non-hospitalized subjects has been reported to be $0.6 \%$ [41] and 1.3\% [42]. The same prevalence of $0.6 \%$ has been reported among Spanish patients [17]. When focusing at species level, studies in Denmark [43], France [19] and Czech Republic [44] showed that respectively $0.06 \%, 0.09 \%$ and $1.3 \%$ of clinical E.coli isolates were pAmpC positive. For K.pneumoniae this was $0.5 \%$ of K.pneumoniae in Czech Republic [44] and 3\% of K.pneumoniae in Ireland [45]. In our study, in Egypt, these percentages were higher: $2,4 \%$ of clinical E.coli isolates

Table 2 Occurence of plasmidal AmpC in group I Enterobacteriaceae

\begin{tabular}{lll}
\hline Population & Proportion of pAmpC+ Enterobacteriaceae & Percentage pAmpC+ $(95 \% \mathrm{Cl})$ \\
\hline $\begin{array}{l}\text { Patients suspected of blood stream infection } \\
(n=225)\end{array}$ & $4 / 72$ group I Enterobacteriaceae & $5,6 \%$ of group I Enterobacteriaceae $(2,2-13,4)$ \\
& $3 / 55$ E.coli & $5,5 \%$ of E.coli $(1,9-14,9)$ \\
& $1 / 15$ K.pneumoniae & $6,7 \%$ of K.pneumoniae $(1,2-29,8)$ \\
Patients with community-onset gastroenteritis & $5 / 105$ group I Enterobacteriaceae & $4,8 \%$ of group I Enterobacteriaceae $(2,1-10,7)$ \\
$(n=100)$ & $0 / 72$ E.coli & $0 \%$ of E.coli $(0-5,1)$ \\
& $3 / 23$ K.pneumoniae & $13 \%$ of K.pneumoniae $(4,5-32,1)$ \\
& $2 / 5$ P.mirabilis & \\
Retail chicken meat & $2 / 83$ group I Enterobacteriaceae \\
$(n=112)$ & $2 / 37$ E.coli & $2,4 \%$ of group I Enterobacteriaceae $(0,7-8,4)$ \\
& $0 / 44$ K.pneumoniae & $5,4 \%$ (1,5-17,7) of E.coli \\
Retail sheep meat & $1 / 95$ group I Enterobacteriaceae & $0 \%$ of K.pneumoniae $(0-8,0)$ \\
$(n=100)$ & $1 / 93$ E.coli & $1,1 \%$ of group I Enterobacteriaceae $(0,2-5,7)$ \\
\hline
\end{tabular}


Table 3 Susceptibility patterns for pAmpC positive group I Enterobacteriaceae

\begin{tabular}{|c|c|c|c|c|c|c|c|c|c|}
\hline $\begin{array}{l}\bar{\pi} \\
\frac{\pi}{2} \\
\stackrel{\pi}{ \pm} \\
\sum\end{array}$ & 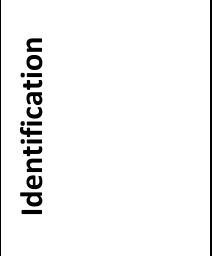 & 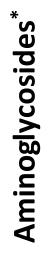 & $\begin{array}{l}\frac{5}{0} \\
\mathbb{x} \\
\frac{0}{4} \\
\frac{0}{4} \\
\frac{0}{0} \\
\frac{0}{3}\end{array}$ & 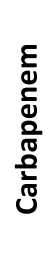 & 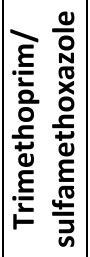 & 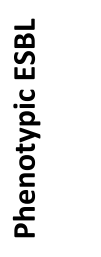 & 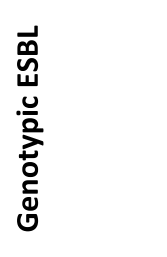 & 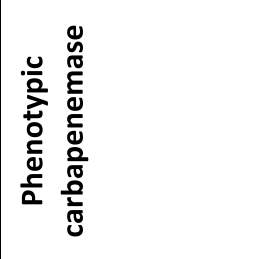 & 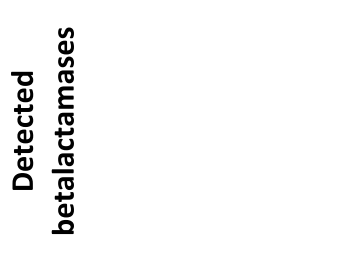 \\
\hline Blood & E.coli & $\mathrm{R}$ & $\mathrm{R}$ & S & $\mathrm{R}$ & $\mathrm{ESBL}+$ & ESBL+ & carbapenemase- & CTX-M-1 \\
\hline Blood & E.coli & $S$ & $\mathrm{R}$ & $\mathrm{S}$ & $S$ & ESBL- & not tested & carbapenemase- & not tested \\
\hline Blood & E.coli & $S$ & $\mathrm{~S}$ & $\mathrm{~S}$ & $\mathrm{R}$ & ESBL+ & ESBL- & carbapenemase- & TEM1 \\
\hline Blood & K.pneumoniae & $S$ & $\mathrm{~S}$ & $\mathrm{~S}$ & $\mathrm{R}$ & ESBL+ & ESBL+ & carbapenemase- & CTX-M-1/SHV/TEM1 \\
\hline Feces & K.pneumoniae & $\mathrm{R}$ & $R$ & $\mathrm{~S}$ & $S$ & ESBL+ & ESBL+ & carbapenemase- & CTX-M-9/SHV \\
\hline Feces & K.pneumoniae & $\mathrm{R}$ & $\mathrm{S}$ & $\mathrm{R}$ & $\mathrm{R}$ & N.I. & ESBL+ & carbapenemase+ & CTX-M-1/SHV/TEM/VIM \\
\hline Feces & K.pneumoniae & $\mathrm{R}$ & $\mathrm{S}$ & $\mathrm{R}$ & $\mathrm{R}$ & N.I. & ESBL+ & carbapenemase+ & CTX-M-1/SHV/TEM/VIM \\
\hline Feces & P.mirabilis & $\mathrm{R}$ & $\mathrm{S}$ & I & $\mathrm{R}$ & ESBL+ & ESBL- & carbapenemase- & None \\
\hline Feces & P.mirabilis & $S$ & $\mathrm{R}$ & I & R & ESBL- & ESBL- & carbapenemase- & None \\
\hline Poultry & E.coli & S & $\mathrm{S}$ & $S$ & $S$ & ESBL- & ESBL- & carbapenemase- & None \\
\hline Poultry & E.coli & $\mathrm{R}$ & $\mathrm{S}$ & $\mathrm{S}$ & $S$ & ESBL- & not tested & carbapenemase- & not tested \\
\hline Sheep & E.coli & $S$ & I & $\mathrm{S}$ & $\mathrm{R}$ & ESBL+ & ESBL+ & carbapenemase- & CTX-M-1,2 or3/TEM \\
\hline
\end{tabular}

$R$ resistant, $S$ susceptible, $/$ intermediate susceptible, N.I. not interpretable, + positive, - negative

Red colour: resistance (mechanism) detected. Green colour: no resistance (mechanism) detected, orange colour: intermediate susceptibility detected. "gentamicin and/or tobramycin

and $10.5 \%$ of clinical K.pneumoniae isolates were pAmpC positive. In Asia, the percentage of clinical E.coli isolates that is $\mathrm{pAmpC}$ positive varies over a wider range. The lowest prevalence has been reported in studies in Japan (0.12\% and $1.7 \%$ of E.coli $[46,47])$, Iran (2.8\% of E.coli [48]) and China (2\% of E.coli $[49,50])$. In a study in Turkey, $10.9 \%$ of clinical E.coli isolates carried pAmpC genes [23]. The highest prevalence was found in India: of the 132 E.coli isolates, that were acquired from unique hospitalized patients in six distant hospitals in India, 60 were positive in the $\mathrm{pAmpC} P C R$, resulting in a prevalence of $45.5 \%$ [51]. In this study, the prevalence among K.pneumoniae was also high (29.4\%), whereas in other studies in Asia the prevalence of $\mathrm{pAmpC}$ among K.pneumoniae was $10 \%$ or less [23, 46, 49, 50].

When evaluating the currently available literature discussed above, the prevalence of pAmpC of around 5\%, as found in our study, is similar to the prevalence as found in other North African countries (ranging from 2.3\%-6.7\%). It should be mentioned, however, that in general, data regarding prevalence of $\mathrm{pAmpC}$ are difficult to compare, since studies use different methods for the detection of pAmpC. First of all, screening methods for detection of suspected pAmpC-producing isolates differ widely. Secondly, PCR-based confirmation methods vary. Many studies used the PCR as initially described by Pérez-Pérez in 2002 [33]. At that time, 29 AmpC genes within 6 $\mathrm{AmpC}$ families were known. In the past few years, however, the number of known AmpC genes has increased. Therefore, in our study, we used a PCR that aimed to detect 183 AmpC genes. Consequently, previous studies might have missed AmpC genes that were not yet discovered at that time or that were not targeted by the used AmpC PCR. Additionally, some of the studies did not aim to detect AmpC genes of all six AmpC families. In some studies only PCR was done for detecting members of the CIT family, or the focus was only on detecting $b l a_{C M Y}$. Furthermore, in some studies the sample sizes were small, resulting in large 95\% confidence intervals. Therefore, there are several limitations when comparing studies on prevalence of pAmpC. Still, we consider the prevalence of pAmpC among Egyptian patients, as found in our study, moderately low. As a reference, we previously showed that prevalence of ESBL among Enterobacteriaceae recovered from patients with community-onset gastroenteritis and blood stream infection in Egypt was much higher: 65\% and $49 \%$ respectively $[28,30]$.

We also determined the prevalence of $\mathrm{pAmpC}$ among Enterobacteriaceae recovered from Egyptian retail meat. We found that between $1 \%$ and $2.5 \%$ of group I Enterobacteriaceae isolated from retail sheep and chicken were pAmpC positive. Compared to other studies, this is low. 
Previous studies have shown a prevalence of $\mathrm{pAmpC}$ in retail chicken of 9-12\% [24-27]. Whether it is likely that retail meat in Egypt forms a reservoir for pAmpC producing Enterobacteriaceae cannot be deducted from our study and needs further investigation. If, however, pAmpC would spread from meat for consumption to humans it will be important to take adequate measures in livestock breeding, such as minimizing consumption of antibiotics in animals and proper food preparation.

Most of the pAmpC positive isolates in our study ( 9 out of 12) contained AmpC genes belonging to the CIT family. This is in concordance with studies all over the world, showing that CMY-2 is the most prevalent pAmpC gene [15, 19, 24, 26, 38, 52, 53]. We found two DHA positive isolates, which have most often been detected in Asia [46, 49]. The less common EBC variant was detected only once in our study. Compared to the study by Helmy et al., which was also conducted in Egypt, genotypic distribution among $\mathrm{pAmpC}$ positive isolates was about the same, although they also detected three genes that belonged to the MOX family and three genes that belonged to the FOX family [38].

It is known that plasmids that encode $\mathrm{AmpC}$ genes often carry many other resistance genes [12, 15, 54, 55]. Indeed, we found that almost half of the plasmid-encoding AmpC Enterobacteriaceae showed resistance to three out of the four most important alternative drug choices for treating infections with pAmpC producing Enterobacteriaceae: aminoglycosides, ciprofloxacin, carbapenems and trimethoprim/sulfamethoxazole. Resistance to trimethoprim/ sulfamethoxazole was seen most often, in 8 of $12 \mathrm{pAmpC}$ positive isolates. Carbapenem resistance was seen in four isolates, in which in two cases it was caused by carbapenemase production. In the other two cases it might have been caused by the concomitance of $\mathrm{pAmpC}$ with porin loss $[5,13,14]$. This co-existence of pAmpC and resistance to other alternative antibiotic therapy is worrying; it is likely to cause therapy failure since few or no therapeutic options are left.

Although the prevalence of pAmpC in Egypt is still relatively low, the occurrence of $\mathrm{pAmpC}$ in humans and in meat for consumption poses a possible threat comparable to ESBL. Currently, international guidelines do not provide recommendations for the detection of $\mathrm{pAmpC}$ in routine laboratory practice. Therefore, in most laboratories, pAmpC producers will not be recognized. This might have consequences for the further spread of pAmpC. Indeed, it has been shown that prevalence of pAmpC has increased over time $[49,56]$. This increase, together with co-existence of resistance to important alternative antibiotic therapies, forms a potential threat. We believe that, for better understanding the occurrence and consequences of $\mathrm{pAmpC}$, standardized screening algorithms for the detection of $\mathrm{pAmpC}$ are required.

\section{Conclusions}

Currently, standardized screening algorithms for the detection of pAmpC are lacking. Therefore studies that describe the prevalence of $\mathrm{pAmpC}$ use different detection methods and consequently are difficult to compare. Based on the current literature, we consider the prevalence of $\mathrm{pAmpC}$ in Egypt, as found in our study, moderately low. In order to be able to follow future trends in the prevalence of pAmpC worldwide, a standardized screening algorithm for the detection of $\mathrm{pAmpC}$ is necessary.

\section{Abbreviations \\ AmpC: AmpC betalactamases; ESBL: Extended-spectrum beta-lactamase; pAmpC: Plasmid-mediated AmpC}

\section{Acknowledgments}

We would like to acknowledge the staff of the microbiology department, El-Ahrar General Hospital, Zagazig, Egypt, for collecting the blood and feces samples. We would like to thank Dr. Robert Roosendaal and dr. Annika Pettersson for their expertise and support in implementing the AmpC PCR.

\section{Funding}

The research was partly funded by the joint supervision mission between Egyptian high education ministry and VU medical center, the Netherlands.

\section{Availability of data and materials}

The authors declare that the data supporting the findings of this study are available within the article.

\section{Authors' contributions}

KLR, HMA, GAE, CMJEV, NaN and KvD made substantial contributions to conception and design of the study. KLR, HMA and AK performed the experiments and analyzed the data. KLR, HMA, GAE, CMJEV, NaN and KvD made substantial contributions to interpretation of the data. KLR wrote the initial draft of the manuscript. HMA, CMJEV, NaN and KvD contributed to critically revising the manuscript. All authors read and approved the final manuscript.

\section{Ethics approval and consent to participate}

All patients with suspected bloodstream infection and gastroenteritis, that were included in this study, provided written informed consent. The human part of this study was approved by the review boards of the Research Ethics Committee, Faculty of Medicine, Zagazig University.

\section{Consent for publication}

Not applicable.

\section{Competing interests}

The authors declare that they have no competing interests.

\section{Publisher's Note}

Springer Nature remains neutral with regard to jurisdictional claims in published maps and institutional affiliations.

\section{Author details}

${ }^{1}$ Amsterdam UMC, Vrije Universiteit Amsterdam, Medical Microbiology and Infection Control, Amsterdam Immunity and Infection Institute, Amsterdam, The Netherlands. ${ }^{2}$ Department of Microbiology, Faculty of Veterinary Medicine, Zagazig University, Zagazig, Egypt. ${ }^{3}$ Laboratory for Medical Microbiology and Public Health, Hengelo, The Netherlands. ${ }^{4}$ Microbiology and Infection Control, Ziekenhuisgroep Twente, Almelo, The Netherlands.

Received: 14 November 2018 Accepted: 4 February 2019

Published online: 26 February 2019

\section{References}

1. World Health Organization. Antimicrobial resistance: global report on surveillance: WHO; 2014. https://www.who.int/drugresistance/documents/ surveillancereport/en/. 
2. Jacoby GA. AmpC beta-lactamases. Clin Microbiol Rev. 2009;22(1):161-82 Table of Contents.

3. Bernards AT, Bonten MJM, Cohen Stuart J, Diederen BMW, Goessens WHF, Grundmann $\mathrm{H}$, et al. NVMM Guideline. Laboratory detection of highly resistant microorganisms (HRMO), version 2.0. 2012; Available at: https:/ www.nvmm.nl/zorg/richtlijnen/nvmm-richtlijnen/.

4. Lister PD, Wolter DJ, Hanson ND. Antibacterial-resistant Pseudomonas aeruginosa: clinical impact and complex regulation of chromosomally encoded resistance mechanisms. Clin Microbiol Rev. 2009;22(4):582-610.

5. Matsumura $Y$, Tanaka M, Yamamoto M, Nagao M, Machida K, Ito $Y$, et al. High prevalence of carbapenem resistance among plasmid-mediated AmpC beta-lactamase-producing Klebsiella pneumoniae during outbreaks in liver transplantation units. Int J Antimicrob Agents. 2015; 45(1):33-40.

6. Arena F, Giani T, Becucci E, Conte V, Zanelli G, D'Andrea MM, et al. Large oligoclonal outbreak due to Klebsiella pneumoniae ST14 and ST26 producing the FOX-7 AmpC beta-lactamase in a neonatal intensive care unit. J Clin Microbiol. 2013;51(12):4067-72.

7. Huang IF, Chiu CH, Wang MH, Wu CY, Hsieh KS, Chiou CC. Outbreak of dysentery associated with ceftriaxone-resistant Shigella sonnei: First report of plasmid-mediated CMY-2-type AmpC beta-lactamase resistance in S. sonnei. J Clin Microbiol. 2005;43(6):2608-12.

8. Ktari S, Arlet G, Verdet C, Jaoua S, Kachrid A, Ben Redjeb S, et al. Molecular epidemiology and genetic environment of acquired bla ACC-1 in Salmonella enterica serotype Livingstone causing a large nosocomial outbreak in Tunisia. Microb Drug Resist. 2009;15(4):279-86.

9. Nadjar D, Rouveau M, Verdet C, Donay L, Herrmann J, Lagrange PH, et al. Outbreak of Klebsiella pneumoniae producing transferable AmpCtype beta-lactamase (ACC-1) originating from Hafnia alvei. FEMS Microbiol Lett. 2000;187(1):35-40.

10. Ohana S, Leflon V, Ronco E, Rottman M, Guillemot D, Lortat-Jacob S, et al. Spread of a Klebsiella pneumoniae strain producing a plasmid-mediated ACC-1 AmpC beta-lactamase in a teaching hospital admitting disabled patients. Antimicrob Agents Chemother. 2005:49(5):2095-7.

11. Roh KH, Uh Y, Kim JS, Kim HS, Shin DH, Song W. First outbreak of multidrug-resistant Klebsiella pneumoniae producing both SHV-12-type extended-spectrum beta-lactamase and DHA-1-type AmpC beta-lactamase at a Korean hospital. Yonsei Med J. 2008:49(1):53-7.

12. Rodriguez-Bano J, Miro E, Villar M, Coelho A, Gozalo M, Borrell N, et al. Colonisation and infection due to Enterobacteriaceae producing plasmidmediated AmpC beta-lactamases. J Infect. 2012;64(2):176-83.

13. Dahmen S, Mansour W, Charfi K, Boujaafar N, Arlet G, Bouallegue O. Imipenem resistance in Klebsiella pneumoniae is associated to the combination of plasmid-mediated CMY-4 AmpC beta-lactamase and loss of an outer membrane protein. Microb Drug Resist. 2012;18(5):479-83.

14. Chiu SK, Wu TL, Chuang YC, Lin JC, Fung CP, Lu PL, et al. National surveillance study on carbapenem non-susceptible Klebsiella pneumoniae in Taiwan: the emergence and rapid dissemination of KPC-2 carbapenemase. PLoS One. 2013;8(7):e69428.

15. Abdalhamid B, Albunayan S, Shaikh A, Elhadi N, Aljindan R. Prevalence study of plasmid-mediated AmpC beta-lactamases in Enterobacteriaceae lacking inducible ampC from Saudi hospitals. J Med Microbiol. 2017;66(9):1286-90.

16. Pai $\mathrm{H}$, Kang $\mathrm{Cl}$, Byeon JH, Lee KD, Park WB, Kim HB, et al. Epidemiology and clinical features of bloodstream infections caused by AmpC-type-betalactamase-producing Klebsiella pneumoniae. Antimicrob Agents Chemother. 2004;48(10):3720-8.

17. Garrido A, Seral C, Gude MJ, Casado C, Gonzalez-Dominguez M, Saenz Y, et al. Characterization of plasmid-mediated beta-lactamases in fecal colonizing patients in the hospital and community setting in Spain. Microb Drug Resist. 2014;20(4):301-4.

18. Miro E, Aguero J, Larrosa MN, Fernandez A, Conejo MC, Bou G, et al. Prevalence and molecular epidemiology of acquired $\mathrm{AmpC}$ beta-lactamases and carbapenemases in Enterobacteriaceae isolates from 35 hospitals in Spain. Eur J Clin Microbiol Infect Dis. 2013;32(2):253-9.

19. Corvec S, Cremet L, Leprince C, Dauvergne S, Reynaud A, Lepelletier D, et al. Epidemiology of Escherichia coli clinical isolates producing AmpC plasmidic beta-lactamase during a 5-year period in a French teaching Hospital. Diagn Microbiol Infect Dis. 2010;67(3):277-81.

20. Ahmed SF, Ali MM, Mohamed ZK, Moussa TA, Klena JD. Fecal carriage of extended-spectrum beta-lactamases and AmpC-producing Escherichia coli in a Libyan community. Ann Clin Microbiol Antimicrob. 2014;13:22.
21. labadene H, Messai Y, Ammari H, Alouache S, Verdet C, Bakour R, et al. Prevalence of plasmid-mediated AmpC beta-lactamases among Enterobacteriaceae in Algiers hospitals. Int J Antimicrob Agents. 2009; 34(4):340-2

22. Barguigua A, El Otmani F, Talmi M, Zerouali K, Timinouni M. Prevalence and types of extended spectrum beta-lactamases among urinary Escherichia coli isolates in Moroccan community. Microb Pathog. 2013;61-62:16-22.

23. Yilmaz NO, Agus N, Bozcal E, Oner O, Uzel A. Detection of plasmidmediated AmpC beta-lactamase in Escherichia coli and Klebsiella pneumoniae. Indian J Med Microbiol. 2013;31(1):53-9.

24. Pehlivanlar Onen S, Aslantas O, Sebnem Yilmaz E, Kurekci C. Prevalence of beta-Lactamase Producing Escherichia coli from Retail Meat in Turkey. J Food Sci. 2015;80(9):M2023-9.

25. Ghodousi A, Bonura C, Di Noto AM, Mammina C. Extended-Spectrum ss-Lactamase, AmpC-Producing, and Fluoroquinolone-Resistant Escherichia coli in Retail Broiler Chicken Meat, Italy. Foodborne Pathog Dis. 2015;12(7):619-25.

26. Maamar E, Hammami S, Alonso CA, Dakhli N, Abbassi MS, Ferjani S, et al. High prevalence of extended-spectrum and plasmidic AmpC betalactamase-producing Escherichia coli from poultry in Tunisia. Int J Food Microbiol. 2016;231:69-75.

27. Voets GM, Fluit AC, Scharringa J, Schapendonk C, van den Munckhof T, Leverstein-van Hall MA, et al. Identical plasmid AmpC beta-lactamase genes and plasmid types in E. coli isolates from patients and poultry meat in the Netherlands. Int J Food Microbiol. 2013;167(3):359-62.

28. Abdallah HM, Alnaiemi N, Reuland EA, Wintermans BB, Koek A, Abdelwahab $\mathrm{AM}$, et al. Fecal carriage of extended-spectrum beta-lactamase- and carbapenemase-producing Enterobacteriaceae in Egyptian patients with community-onset gastrointestinal complaints: a hospital -based crosssectional study. Antimicrob Resist Infect Control. 2017;6:62.

29. Abdallah HM, Reuland EA, Wintermans BB, Al Naiemi N, Koek A, Abdelwahab AM, et al. Extended-Spectrum beta-Lactamases and/or Carbapenemases-Producing Enterobacteriaceae Isolated from Retail Chicken Meat in Zagazig, Egypt. PLoS One. 2015;10(8):e0136052.

30. Abdallah HM, Wintermans BB, Reuland EA, Koek A, al Naiemi N, Ammar AM, et al. Extended-Spectrum beta-Lactamase- and Carbapenemase-Producing Enterobacteriaceae Isolated from Egyptian Patients with Suspected Blood Stream Infection. PLoS One. 2015;10(5):e0128120.

31. Pearce RA, Bolton DJ. Excision vs sponge swabbing - a comparison of methods for the microbiological sampling of beef, pork and lamb carcasses. J Appl Microbiol. 2005;98(4):896-900.

32. Brolund A, Wisell KT, Edquist PJ, Elfstrom L, Walder M, Giske CG. Development of a real-time SYBRGreen PCR assay for rapid detection of acquired AmpC in Enterobacteriaceae. J Microbiol Methods. 2010;82(3):229-33.

33. Perez-Perez FJ, Hanson ND. Detection of plasmid-mediated AmpC betalactamase genes in clinical isolates by using multiplex PCR. J Clin Microbiol. 2002;40(6):2153-62.

34. EUCAST European Committee on Antimicrobial Susceptibility Testing Breakpoint tables for interpretation of MICs and zone diameters Version 8.1, 2018. http://www.eucast.org

35. EUCAST guideline for the detection of resistance mechanisms and specific resistances of clinical and/or epidemiological importance. version 2.0, 2017. http://www.eucast.org/resistance mechanisms.

36. Pasteran F, Mendez T, Guerriero L, Rapoport M, Corso A. Sensitive screening tests for suspected class A carbapenemase production in species of Enterobacteriaceae. J Clin Microbiol. 2009;47(6):1631-9.

37. Tsakris A, Kristo I, Poulou A, Themeli-Digalaki K, Ikonomidis A, Petropoulou D, et al. Evaluation of boronic acid disk tests for differentiating KPCpossessing Klebsiella pneumoniae isolates in the clinical laboratory. J Clin Microbiol. 2009:47(2):362-7.

38. Helmy MM, Wasfi R. Phenotypic and molecular characterization of plasmid mediated AmpC beta-lactamases among Escherichia coli, Klebsiella spp., and Proteus mirabilis isolated from urinary tract infections in Egyptian hospitals. Biomed Res Int. 2014;2014:171548.

39. Park YS, Yoo S, Seo MR, Kim JY, Cho YK, Pai H. Risk factors and clinical features of infections caused by plasmid-mediated AmpC betalactamase-producing Enterobacteriaceae. Int J Antimicrob Agents. 2009; 34(1):38-43.

40. Bassyouni RH, Gaber SN, Wegdan AA. Fecal carriage of extended-spectrum beta-lactamase- and AmpC-producing Escherichia coli among healthcare workers. J Infect Dev Ctries. 2015;9(3):304-8. 
41. van Hoek AH, Schouls L, van Santen MG, Florijn A, de Greeff SC, van Duijkeren E. Molecular characteristics of extended-spectrum cephalosporin-resistant Enterobacteriaceae from humans in the community. PLoS One. 2015;10(6):e0129085.

42. Reuland EA, Halaby T, Hays JP, de Jongh DM, Snetselaar HD, van Keulen M, et al. Plasmid-mediated AmpC: prevalence in community-acquired isolates in Amsterdam, the Netherlands, and risk factors for carriage. PLoS One. 2015:10(1):e0113033.

43. Jorgensen RL, Nielsen JB, Friis-Moller A, Fjeldsoe-Nielsen H, Schonning K. Prevalence and molecular characterization of clinical isolates of Escherichia coli expressing an AmpC phenotype. J Antimicrob Chemother. 2010;65(3):460-4.

44. Husickova V, Chroma M, Kolar M, Hricova K, Stosova T, Kantor L, et al. Analysis of ESBL- and AmpC-positive Enterobacteriaceae at the Department of Neonatology, University Hospital Olomouc. Curr Microbiol. 2011;62(6):1664-70.

45. Roche C, Boo TW, Walsh F, Crowley B. Detection and molecular characterisation of plasmidic AmpC beta-lactamases in Klebsiella pneumoniae isolates from a tertiary-care hospital in Dublin, Ireland. Clin Microbiol Infect. 2008:14(6):616-8

46. Yamasaki K, Komatsu M, Abe N, Fukuda S, Miyamoto Y, Higuchi T, et al. Laboratory surveillance for prospective plasmid-mediated AmpC betalactamases in the Kinki region of Japan. J Clin Microbiol. 2010;48(9):3267-73.

47. Matsumura $Y$, Yamamoto $M$, Higuchi $T$, Komori T, Tsuboi F, Hayashi A, et al. Prevalence of plasmid-mediated AmpC beta-lactamase-producing Escherichia coli and spread of the ST131 clone among extendedspectrum beta-lactamase-producing E. coli in Japan. Int J Antimicrob Agents. 2012;40(2):158-62.

48. Shayan S, Bokaeian M, Shahraki S. Prevalence and molecular characterization of AmpC-producing clinical isolates of Escherichia coli from southeastern Iran. Microb Drug Resist. 2014;20(2):104-7.

49. Ding $H$, Yang $Y$, Lu $Q$, Wang $Y$, Chen $Y$, Deng $L$, et al. The prevalence of plasmid-mediated AmpC beta-lactamases among clinical isolates of Escherichia coli and Klebsiella pneumoniae from five children's hospitals in China. Eur J Clin Microbiol Infect Dis. 2008;27(10):915-21.

50. Li Y, Li Q, Du Y, Jiang X, Tang J, Wang J, et al. Prevalence of plasmidmediated AmpC beta-lactamases in a Chinese university hospital from 2003 to 2005: first report of CMY-2-Type AmpC beta-lactamase resistance in China. J Clin Microbiol. 2008;46(4):1317-21.

51. Mohamudha PR, Harish BN, Parija SC. Molecular description of plasmidmediated AmpC beta-lactamases among nosocomial isolates of Escherichia coli \& Klebsiella pneumoniae from six different hospitals in India. Indian J Med Res. 2012;135:114-9.

52. Denisuik AJ, Lagace-Wiens PR, Pitout JD, Mulvey MR, Simner PJ, Tailor F, et al. Molecular epidemiology of extended-spectrum beta-lactamase-, AmpC betalactamase- and carbapenemase-producing Escherichia coli and Klebsiella pneumoniae isolated from Canadian hospitals over a 5 year period: CANWARD 2007-11. J Antimicrob Chemother. 2013;68(Suppl 1):57-65.

53. Gude MJ, Seral C, Saenz Y, Cebollada R, Gonzalez-Dominguez M, Torres C, et al. Molecular epidemiology, resistance profiles and clinical features in clinical plasmid-mediated AmpC-producing Enterobacteriaceae. Int J Med Microbiol. 2013;303(8):553-7.

54. Philippon A, Arlet G, Jacoby GA. Plasmid-determined AmpC-type betalactamases. Antimicrob Agents Chemother. 2002:46(1):1-11.

55. Mata C, Miro E, Rivera A, Mirelis B, Coll P, Navarro F. Prevalence of acquired AmpC beta-lactamases in Enterobacteriaceae lacking inducible chromosomal ampC genes at a Spanish hospital from 1999 to 2007. Clin Microbiol Infect. 2010;16(5):472-6.

56. Wang JT, Chang SC, Chang FY, Fung CP, Chuang YC, Chen YS, et al. Antimicrobial Non-Susceptibility of Escherichia coli from Outpatients and Patients Visiting Emergency Rooms in Taiwan. PloS one. 2015; 10(12):e0144103.

Ready to submit your research? Choose BMC and benefit from:

- fast, convenient online submission

- thorough peer review by experienced researchers in your field

- rapid publication on acceptance

- support for research data, including large and complex data types

- gold Open Access which fosters wider collaboration and increased citations

- maximum visibility for your research: over $100 \mathrm{M}$ website views per year

At BMC, research is always in progress.

Learn more biomedcentral.com/submissions 